ARTICLE

https://doi.org/10.1038/s41467-019-10273-2

\title{
Scaling growth rates for perovskite oxide virtual substrates on silicon
}

\author{
Jason Lapano ${ }^{1}$, Matthew Brahlek ${ }^{1,5}$, Lei Zhang (1D ${ }^{2}$, Joseph Roth ${ }^{1}$, Alexej Pogrebnyakov ${ }^{1}$ \& \\ Roman Engel-Herbert ${ }^{1,3,4}$
}

The availability of native substrates is a cornerstone in the development of microelectronic technologies relying on epitaxial films. If native substrates are not available, virtual substrates - crystalline buffer layers epitaxially grown on a structurally dissimilar substrate - offer a solution. Realizing commercially viable virtual substrates requires the growth of high-quality films at high growth rates for large-scale production. We report the stoichiometric growth of $\mathrm{SrTiO}_{3}$ exceeding $600 \mathrm{~nm} \mathrm{hr}^{-1}$. This tenfold increase in growth rate compared to $\mathrm{SrTiO}_{3}$ grown on silicon by conventional methods is enabled by a self-regulated growth window accessible in hybrid molecular beam epitaxy. Overcoming the materials integration challenge for complex oxides on silicon using virtual substrates opens a path to develop new electronic devices in the More than Moore era and silicon integrated quantum computation hardware.

\footnotetext{
${ }^{1}$ Department of Materials Science and Engineering, Pennsylvania State University, University Park, PA 16802, USA. ${ }^{2}$ Department of Materials Science and Engineering, University of California, Berkeley, CA 94720, USA. ${ }^{3}$ Department of Physics, Pennsylvania State University, University Park, PA 16802, USA.

${ }^{4}$ Department of Chemistry, Pennsylvania State University, University Park, PA 16802, USA. ${ }^{5}$ Present address: Materials Science and Technology, Oak Ridge National Lab, Oak Ridge, TN 37831, USA. Correspondence and requests for materials should be addressed to R.E.-H. (email: rue2@psu.edu)
} 
S uccessful implementation of epitaxial thin film technologies hinges upon the availability of an economical single-crystal substrate. These substrates must be both chemically and structurally compatible with the desired film to prevent unwanted interfacial defect formation, and must be economically feasible for large-scale production. When no native single crystalline substrate that satisfies these requirements is available, virtual substrates, i.e., the growth of buffer layers allowing a change in lattice parameter, structure or even chemistry of the available substrate, can provide a solution. In such cases robust and cost effective, while scalable, material integration schemes that suffice stringent economic requirements are in demand to realize new device generations with improved performance at lower cost, weight, and size. Such metamorphic epitaxial materials also represent a route to expand the application space of existing devices, and to realize completely new technologies by stabilizing material phases with otherwise unattainable properties. The technical challenges, such as scalability, high-throughput ${ }^{1}$ and compatibility of the individual fabrication steps to exploit such advancements and deploy them into the market impose stringent conditions for the entire materials integration process.

Metamorphic buffer layers have proven their usefulness at an industrial scale for SiGe, III-V compound semiconductors, and group III-nitrides, setting record performance in a wide application space from photovoltaics using a metamorphic multijunction solar cell with efficiencies up to $46 \%{ }^{2-4}$, III-V based lasers monolithically integrated on $\mathrm{Si}^{5,6}$, high-electron mobility transistors $^{7-9}$, and heterojunction tunnel field effect transistors for highperformance low-power logic ${ }^{10}$. Metamorphic epitaxial materials have allowed for more cost effective and larger non-native substrates for electronic devices ${ }^{11,12}$, and are being considered key to realize and advance the existing quantum computing materials platform of Si/SiGe heterostructures towards all-electrical control of Si-based qubits ${ }^{13}$.

A metamorphic buffer technology for complex perovskite oxides on $\mathrm{Si}$ is highly desirable given their wide range of properties, which can even be expanded utilizing epitaxial strain ${ }^{14}$. Although epitaxial integration of $\mathrm{SrTiO}_{3}$ on $\mathrm{Si}$ has been successfully demonstrated ${ }^{15}$ and has been scaled up to $200 \mathrm{~mm} \mathrm{Si}$ wafers using an industry-scale molecular beam epitaxy (MBE) system ${ }^{16}$, typical film growth rates of about $50 \mathrm{~nm} \mathrm{~h}^{-1}$ or lower ${ }^{16-19}$ impede high-throughput required for profitability. MBE systems have been operated on an industrial scale since the $1980 s^{20}$ primarily for the growth of high-quality binary semiconductor structures with controlled stoichiometry and desired composition at growth rates in excess of $1000 \mathrm{~nm} \mathrm{~h}^{-121,22}$. Metamorphic buffers have been developed for nonoxide materials using this growth technology 23,24 . The key feature permitting such high-quality material by MBE is the adsorption-controlled growth kinetics. The more volatile element is supplied in excess and desorbs in a self-regulated fashion if not incorporated into the film, which dramatically simplifies the flux calibration and enhances reproducibility of the growth process ${ }^{25,26}$. Therefore, the roadblock to integrate perovskites oxide materials by MBE or any other thin film growth technique in a scalable, economic way is the lack of an adsorption-controlled growth mechanism at high film growth rate. Specifically in the case of $\mathrm{SrTiO}_{3}$ deviations from ideal stoichiometry result in a lattice parameter expansion to accommodate defects formed in the film when grown under nonstoichiometric conditions ${ }^{17,27,28}$.

Commonly employed thin film growth techniques can in principle be scaled up to achieve wafer size conformity for complex perovskite oxides and has been demonstrated for pulsed laser deposition (PLD) ${ }^{29}$, sputtering, metalorganic chemical vapor deposition ${ }^{30}$, atomic layer deposition, and $\mathrm{MBE}^{16}$. However, scaling up growth rates have resulted in nonstoichiometric

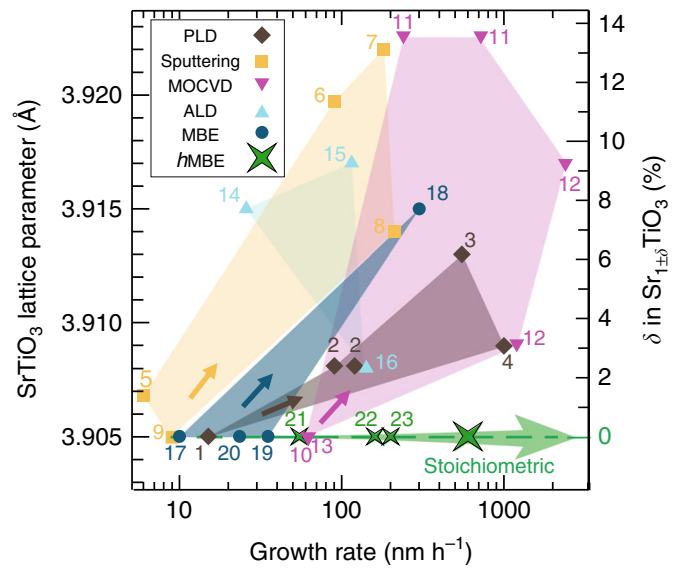

Fig. 1 Growth rates and control of film stoichiometry. Intrinsic $\mathrm{SrTiO}_{3}$ film lattice parameter reported for different growth rates using scalable oxide thin film growth techniques: pulsed laser deposition (PLD), sputtering, metal-organic chemical vapor deposition (MOCVD), atomic layer deposition (ALD), molecular beam epitaxy (MBE), and hybrid molecular beam epitaxy ( $h \mathrm{MBE}$ ). In all cases except hybrid MBE the degree of film nonstoichiometry increased with growth rate. The number close to data points shown refer to references detailed in Supplementary Fig. 1. The defect concentration $\delta$ of $\mathrm{Sr}$-rich $\left(\mathrm{Sr}_{1+\delta} \mathrm{TiO}_{3}\right)$ and Ti-rich $\left(\mathrm{Sr}_{1-\delta} \mathrm{TiO}_{3}\right)$ films due to nonstoichiometric growth condition was determined from the intrinsic film lattice parameter expansion using a calibration curve given in Supplementary Fig. 2. A growth rate of $0.6 \mu \mathrm{m} \mathrm{h}^{-1}$ for stoichiometric $\mathrm{SrTiO}_{3}$ by hybrid MBE was achieved for the growth on LSAT substrates

$\mathrm{SrTiO}_{3}$ films. Figure 1 compiles the degree of stoichiometry control, quantified by the intrinsic $\mathrm{SrTiO}_{3}$ film lattice parameter expansion, as a function of growth rate for different thin film growth methods. Further details can be found in Supplementary Figs. 1 and 2. Sputtering and PLD utilize targets where the indirect control of deposition parameters makes stoichiometry control more challenging with increasing film growth rate. Conventional solid-source MBE growth for perovskite oxides requires precise calibration of the $\mathrm{Sr}$ and $\mathrm{Ti}$ fluxes, however, the level of oxygen pressure needed for high-growth rates adversely affect effusion cell flux stability due to unintentional oxidation of the source material in the crucible ${ }^{31}$, thus limiting this technique to low growth rates ${ }^{16,17}$ and precluding it as a cost-effective production tool for virtual perovskite oxide substrates ${ }^{17,32}$.

We report that the adsorption-controlled growth of $\mathrm{SrTiO}_{3}$ films by hybrid MBE is scaled to growth rates in excess of $600 \mathrm{~nm}$ $\mathrm{h}^{-1}$ with no degradation in structural film quality, rivaling the MBE growth rates commonly employed in industry for the growth of semiconductor thin films. This order of magnitude increase is achieved by co-supplying $\mathrm{Sr}$ from a conventional effusion cell and titanium-tetra-isopropoxide (TTIP) as titanium and oxygen source ${ }^{25,33}$. At such high-growth rates the selfregulated growth window, i.e., the range of TTIP pressures for a given constant $\mathrm{Sr}$ flux at which the film lattice parameter does not expand to accommodate unintentional incorporation of excess cations, remains accessible, which is mapped using in situ reflection high-energy electron-diffraction (RHEED) and confirmed by ex situ X-ray diffraction (XRD) measurements, atomic force microscopy (AFM), and scanning transmission electron microscopy (STEM). This record high-growth rate demonstrated experimentally is merely limited by size and stability of the Sr effusion cell used in the research MBE reactor. The trend extrapolated from the data projects that growth rates of about $9 \mu \mathrm{mh}^{-1}$ are possible if industrial scale MBE hardware is employed instead. 


\section{Results}

Mapping the stoichiometric growth window at high-growth rate. The conditions to access the self-regulated growth of stoichiometric $\mathrm{SrTiO}_{3}$ were determined for a range of growth rates by observing specific surface reconstructions in real time using in situ RHEED ${ }^{34,35}$. This method avoids multiple film growths and time consuming lattice parameter measurements using ex situ XRD ${ }^{36,37}$, and makes accessing the self-regulated growth window a production-line compatible process. This accelerated mapping of growth conditions is key to ensure proper growth condition in a single calibration run in a timely manner, as shown in Fig. 2. In this method, a $\mathrm{SrTiO}_{3}$ film was grown on a $\left(\mathrm{La}_{0.18} \mathrm{Sr}_{0.82}\right)\left(\mathrm{Al}_{0.59} \mathrm{Ta}_{0.41}\right) \mathrm{O}_{3}$ (LSAT) single-crystal substrate. The Sr flux supplied from the effusion cell was held constant at $2.50 \times$ $10^{13} \mathrm{~cm}^{-2} \mathrm{~s}^{-1}$ during the entire calibration run, measured using a quartz crystal monitor at the sample position prior to growth. The TTIP flux, given by the beam equivalent pressure (BEP) $p_{\text {TTIP }}$ measured at the sample position using an ion gauge, was altered throughout the growth by changing the gas inlet pressure. The color code in Fig. 2a indicates whether stoichiometric (green), Srrich (blue), or Ti-rich (purple) growth condition were present. Stoichiometric growth conditions were initially chosen and $\mathrm{Sr}$ and TTIP were co-supplied for $10 \mathrm{~min}$, resulting in a $12 \mathrm{~nm}$-thick template layer. The TTIP flux was then increased and growth was continued for $5 \mathrm{~min}$. This procedure was repeated and RHEED images were taken in real time. Figure $2 \mathrm{a}, \mathrm{b}$ shows the growth sequence along with atomic-resolution STEM micrographs of the calibration sample in cross-section, respectively. STEM imaging revealed that for layers grown under Ti-rich growth conditions small $\mathrm{Ti}$ excess was incorporated as point defects, which gave rise to a gradual increase in background intensity relative to the stoichiometric regions of the calibration sample. For growth conditions with larger $\mathrm{Ti}$ excess the formation of a Ti-rich second phase was observed. In the case of $\mathrm{Sr}$-rich growth conditions
STEM images revealed the local formation of $\mathrm{SrO}$ Ruddlesden-Popper stacking faults propagating throughout the film. $\mathrm{SrTiO}_{3}$ layers were grown within the self-regulated window subsequently on both types of nonstoichiometric layers to determine the minimum thickness required to outgrow the local nonstoichiometry. While stoichiometric $\mathrm{SrTiO}_{3}$ could be repeatedly achieved on layers grown under Ti-rich conditions irrespective of its thickness and degree of Ti-excess, the thickness needed to recover a stoichiometric growth front on layers grown under Sr-rich conditions was about $15 \mathrm{~nm}$. The STEM images further showed that defects from Ti-rich and Sr-rich regions propagated up to about $5 \mathrm{~nm}$ into the subsequently grown layer, shown by regions of higher intensity protruding from the fifth layer into the subsequently grown stoichiometric layer shown in Fig. 2b. This marks the minimum growth duration needed to ensure that growth conditions were accurately assessed by surface sensitive RHEED and subsequent layers were not influenced by potential nonstoichiometries of the preceding layer. The RHEED pattern taken in situ during iterative deposition cycles are shown in Fig. 2c, and in more detail in the Supplementary Fig. 3. In the Sr-rich regime, the RHEED pattern was diffuse with a 2 -fold reconstruction along the 110 azimuth, which can be easily distinguished from the stoichiometric growth conditions, characterized by a sharp RHEED pattern and loss of the 2-fold reconstruction along 110 as well as the appearance of a 2 -fold reconstruction along the 100 . For Ti-rich conditions RHEED patterns were diffuse and exhibited a gradual loss of the 2-fold reconstruction along the $110^{35,36,38}$. Therefore, the growth conditions correlated with the surface reconstruction seen in RHEED and can be used to map the self-regulated growth window on a single calibration sample. The entire growth calibration procedure contained ten different TTIP fluxes and a recovery growth ("reset" layer) after mapping the edge of the growth window towards the Ti-rich growth conditions. The growth of the a

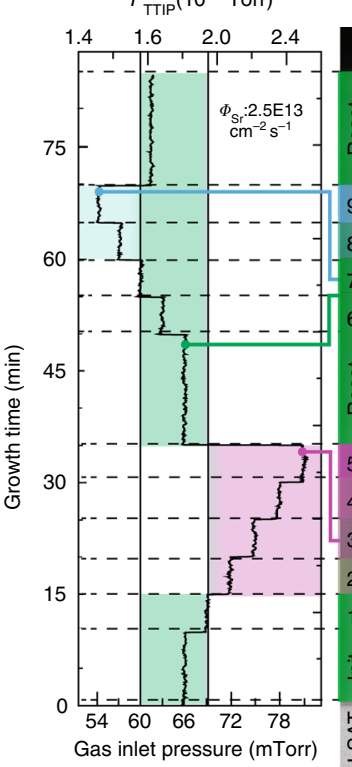

Sr- $-\rightarrow$ Ti-rich b

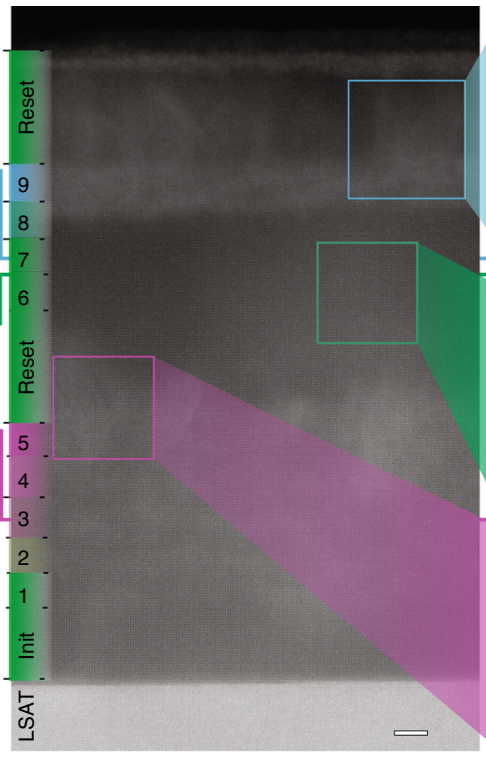

C
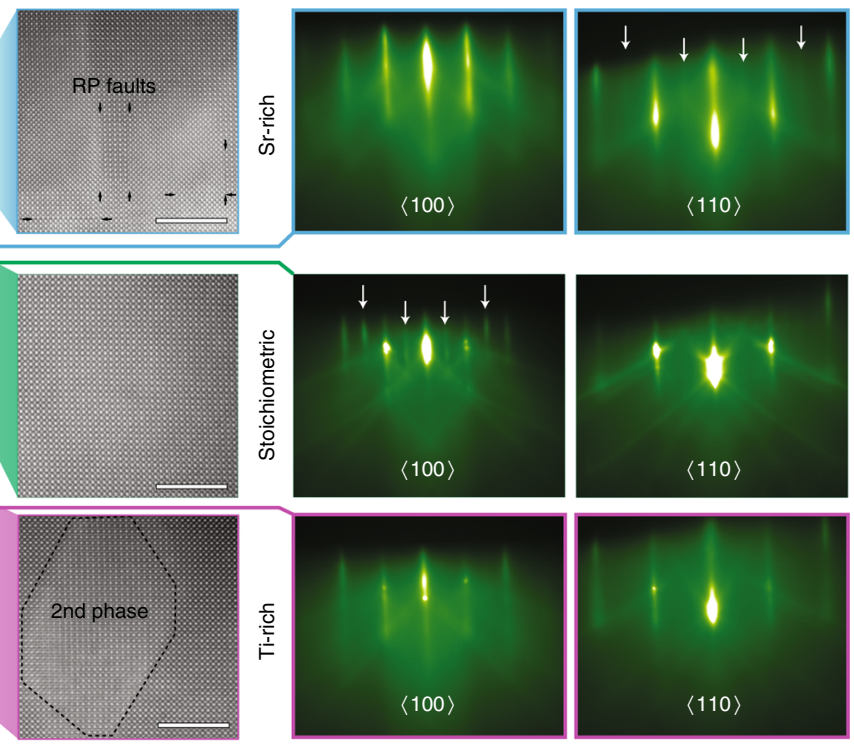

Fig. 2 Mapping growth conditions using production-line compatible process. Calibration sample using a fixed $\mathrm{Sr}$ flux of $2.50 \times 10^{13} \mathrm{~cm}^{-2} \mathrm{~s}^{-1} \mathrm{grown}$ on $\left(\mathrm{La}_{0.18} \mathrm{Sr}_{0.82}\right)\left(\mathrm{Al}_{0.59} \mathrm{Ta}_{0.41}\right) \mathrm{O}_{3}$ (LSAT). a Titanium tetraisopropoxide (TTIP) flux sequence using the beam equivalent pressure $p_{\mathrm{TTIP}}$ as flux measure. $\mathbf{b}$ Highangle annular dark-field scanning transmission electron microscopy (HAADF-STEM) cross-section images of the calibration sample. A higher background intensity was found for layers grown under nonstoichiometric condition. The magnified images illustrate the type of defect formed and how far they protruded into the subsequent layer grown under stoichiometric conditions. The precise position of the Ruddlesden Popper (RP) faults indicated by the arrows were deduced from strain maps shown in Supplementary Fig. 9. c Reflection high-energy electron diffraction (RHEED) images taken in real-time during growth along the $\langle 100\rangle$ and $\langle 110\rangle$ azimuth reflecting the stoichiometry of the growth front. Scale bars on the HAADF images are $5 \mathrm{~nm}$ 

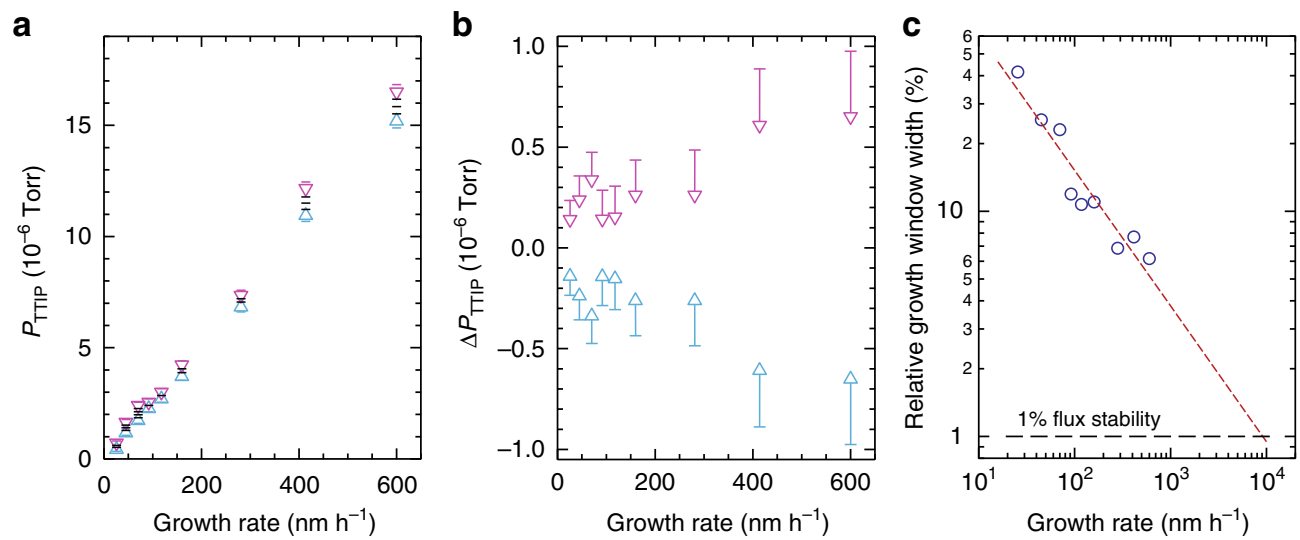

Fig. 3 Scaling growth window. a Titanium tetraisopropoxide (TTIP) beam equivalent pressures $\mathrm{p}_{\text {TTIP }}$ as a function of film growth rate for which a growth window was accessed. The Sr flux was kept fixed while the TTIP flux was modulated. The black bars represent $p_{\text {TTIP }}$ values within the growth window, while the upper red and lower blue triangle indicate the edges of the growth window determined by reflection high-energy electron diffraction (RHEED). The red and blue bar above and below the respective triangles are the first $p_{\mathrm{TTIP}}$ values for Ti-rich and Sr-rich growth conditions, respectively. $\mathbf{b}$ Absolute growth window width $\Delta p_{\text {TTIP }}$ as a function of growth rate. The triangles mark the edges of the growth window, while the bars are the values at which a nonstoichiometric growth front was observed in RHEED. c Relative growth window width $\Delta p_{\text {TTIP }} / p_{\text {TTIP }}$ as a function of growth rate, where $p_{\text {TTIP }}$ is the TTIP beam equivalent pressures at the growth window center. The line is extrapolated based on the existing data set, projecting that a growth rate of (9.1 \pm 1.6$) \mu \mathrm{m} \mathrm{h}^{-1}$ can be achieved at a given Sr flux stability of $1 \%$

calibration sample took about $70 \mathrm{~min}$, which is considerably shortened with increasing growth rate.

Accelerated growth rate of $\mathrm{SrTiO}_{3}$. Rapid growth condition mapping as shown in Fig. 2 was applied to find the self-regulated growth window for Sr-fluxes ranging from $1.25 \times 10^{13} \mathrm{~cm}^{-2} \mathrm{~s}^{-1}$ up to $2.50 \times 10^{14} \mathrm{~cm}^{-2} \mathrm{~s}^{-1}$. The width and position of the growth window as a function of growth rate is shown in Fig. 3. For a $\mathrm{Sr}$ flux of $1.25 \times 10^{13} \mathrm{~cm}^{-2} \mathrm{~s}^{-1}$ (growth rate $25.6 \mathrm{~nm} \mathrm{~h}^{-1}$ ), the TTIP BEP at the growth position had to be kept between 4.28 and $7.10 \times 10^{-7}$ Torr to maintain self-regulated growth, which shifted to $1.52-1.65 \times 10^{-5}$ Torr as the Sr flux was increased to $2.50 \times$ $10^{14} \mathrm{~cm}^{-2} \mathrm{~s}^{-1}$ (growth rate $600.5 \mathrm{~nm} \mathrm{~h}^{-1}$ ) supplied from two $\mathrm{Sr}$ effusion cells operated in tandem. The actual position of the growth window changed linearly with growth rate, which was determined by the Sr flux supplied. The calibration curve of TTIP BEP $p_{\text {TTIP }}$ and gas inlet pressure is shown in Supplementary Fig. 4, while the change in growth rate with supplied Sr flux is shown in Supplementary Fig. 5. The absolute width of the growth window $\Delta p_{\text {TTIP }}$ increased at higher growth rates (Fig. 3b), whereas the relative width $\Delta p_{\text {TTIP }} / p_{\text {TTIP }}$ (Fig. 3c) decreased. The latter trend can be extrapolated to determine the growth rate at which unintentional drifts from the $\mathrm{Sr}$ effusion cell can still be compensated via the self-regulated adsorption mechanism. Assuming a Sr flux drift of about $1 \%$ the growth window would be sufficiently wide up to a growth rate of $(9.1 \pm 1.6) \mu \mathrm{m} \mathrm{h}^{-1}$, as shown in Fig. 3c. This growth rate does not mark the highest possible growth rate that can be achieved using hybrid MBE, since the growth window width has been found to increase with growth temperature ${ }^{26}$.

To confirm stoichiometric growth conditions from film lattice parameter and surface morphology measurements using ex situ XRD and AFM, nominally 45-nm-thick $\mathrm{SrTiO}_{3}$ films were grown on LSAT(100) substrates. Films grown within the growth window showed a step-like terrace structure, a root mean square surface roughness of less than $1 \mathrm{~nm}$ and absence of crystalline islands irrespective of growth rate (Supplementary Fig. 6). Figure 4a shows high-resolution $2 \theta-\omega$ X-ray scans around the $002 \mathrm{SrTiO}_{3}$ film and LSAT substrate peaks. The out-of-plane lattice parameter was found to be $3.930 \pm 0.003 \AA$ irrespective of growth rate, which matches values previously reported for stoichiometric
$\mathrm{SrTiO}_{3}$ films grown on $\mathrm{LSAT}^{39}$. While critical film thickness of $\mathrm{SrTiO}_{3}$ on LSAT substrates was only $180 \mathrm{~nm}^{40}$, a $~ 500-\mathrm{nm}$ thick $\mathrm{SrTiO}_{3}$ film was grown homoepitaxially within the growth window for a growth rate of $600 \mathrm{~nm} \mathrm{~h}^{-1} .2 \theta-\omega \mathrm{X}$-ray scans of the $002 \mathrm{SrTiO}_{3}$ film and substrate peak shown in Fig. $4 \mathrm{~b}$ revealed that they are indistinguishable, as expected for a homoepitaxially grown stoichiometric film ${ }^{41}$. RHEED intensity oscillations are shown in Fig. 4c, d for $\mathrm{SrTiO}_{3}$ films grown at 40 and $600 \mathrm{~nm} \mathrm{~h}^{-1}$ on LSAT, respectively. In both film growths RHEED intensity oscillations were visible which dampened out with time, indicating an initial layer-by-layer growth which transitioned into a step-flow growth mode. The growth of 10 monolayers of $\mathrm{SrTiO}_{3}$ took only $23.8 \mathrm{~s}$, in excellent agreement with the growth rate determined from film thickness measurements. Details of the $\mathrm{X}$-ray fits to determine physical film thickness and intrinsic film lattice parameter are detailed in Supplementary Fig. 7.

Integration on silicon for virtual substrates. To directly prove the compatibility of high $\mathrm{SrTiO}_{3}$ film growth rates with the integration on $\mathrm{Si}$ wafers 125 -nm-thick $\mathrm{SrTiO}_{3}$ thin films were grown and the results are shown in Fig. 5. Two different $\mathrm{Sr}$ sources were used in tandem; a Sr effusion cell with low flux $\left(2.50 \times 10^{13} \mathrm{~cm}^{-2} \mathrm{~s}^{-1}\right)$ to nucleate and initialize the growth on Si using a buffer layer ${ }^{37}$, while a second $\mathrm{Sr}$ cell calibrated to a much higher flux $\left(1.00 \times 10^{14} \mathrm{~cm}^{-2} \mathrm{~s}^{-1}\right)$ for the film growth at a high rate of $240 \mathrm{~nm} \mathrm{~h}^{-1}$. The film thickness was chosen to avoid film cracking due to the thermal mismatch between $\mathrm{Si}$ and $\mathrm{SrTiO}_{3}$. Wide range XRD scans confirmed single crystalline (001) oriented $\mathrm{SrTiO}_{3}$ films with an out-of-plane lattice parameter of $3.889 \AA$, consistent with stoichiometric $\mathrm{SrTiO}_{3}$ films grown on $\mathrm{Si}$ at $850{ }^{\circ} \mathrm{C}$, albeit at much lower growth rates of only $50 \mathrm{~nm} \mathrm{~h}^{-135,37}$. Figure 5b, c shows the rocking curve of the 002 $\mathrm{SrTiO}_{3}$ film peak with a full width at half maximum of $0.28^{\circ}$ along with the reciprocal space map of $\mathrm{SrTiO}_{3} 103$ in excellent agreeement with those reports ${ }^{35,37}$. Figure $5 \mathrm{~d}$ shows the film surface morphology of $\mathrm{SrTiO}_{3}$ on $\mathrm{Si}$ grown at $240 \mathrm{~nm} \mathrm{~h}^{-1}$, which showed a stepped terrace morphology with a root mean square surface roughness value less than $1 \mathrm{~nm}$, making it an ideal starting surface for subsequent epitaxial growth, thus fulfilling yet another requirement of a metamorphic buffer layer. Excellent film thickness uniformity across the entire 3 -in wafer was evident 

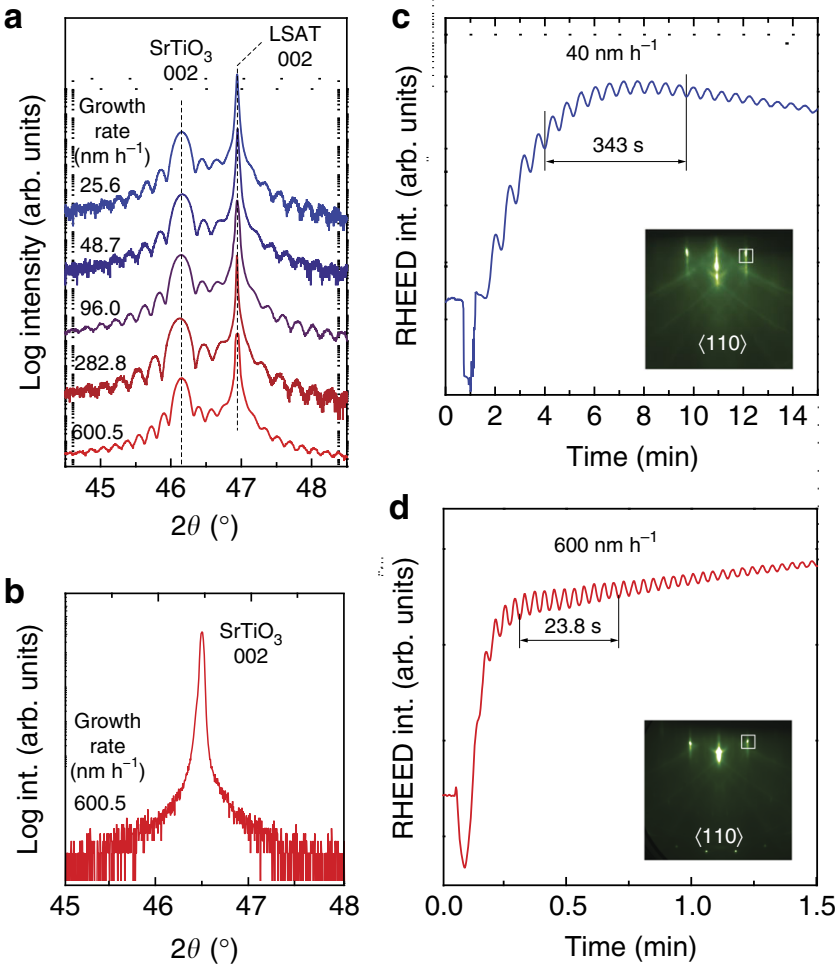

Fig. $4 \mathrm{X}$-ray diffraction and RHEED oscillation. a High resolution $2 \theta-\omega$ X-ray scans of the $002\left(\mathrm{La}_{0.18} \mathrm{Sr}_{0.82}\right)\left(\mathrm{Al}_{0.59} \mathrm{Ta}_{0.41}\right) \mathrm{O}_{3}$ (LSAT) substrate $\left(\sim 46.9^{\circ}\right)$ and $\mathrm{SrTiO}_{3}$ film peaks $\left(46.2^{\circ}\right)$ for growth rates ranging from 25.6 to $600.5 \mathrm{~nm} \mathrm{~h}^{-1}$. b $2 \theta-\omega \mathrm{X}$-ray scan of the 002 peak for a $500-\mathrm{nm}$-thick $\mathrm{SrTiO}_{3}$ film grown homoepitaxially at $600 \mathrm{~nm} \mathrm{~h}^{-1}$. c, d Reflection highenergy electron diffraction (RHEED) intensity oscillation of the 10 diffraction streak (see inset) for $\mathrm{SrTiO}_{3}$ films grown on LSAT at 40 and $600 \mathrm{~nm} \mathrm{~h}^{-1}$. The sharp RHEED intensity drop is caused by the sample shutter operation needed to start the growth, blocking the electron beam

from optical inspection (Fig. 5e) and the $\mathrm{SrTiO}_{3}$ film thickness variation was determined across the entire wafer using spectroscopic ellipsometry, see Fig. 5 f. A less than $1 \%$ change in $\mathrm{SrTiO}_{3}$ thickness was extracted using an optical model detailed in Supplementary Fig. 8. Cross-sectional STEM and energy dispersive spectroscopy in Supplementary Fig. 10 show a single-crystalline $\mathrm{SrTiO}_{3}$ film on the silicon substrate, separated by a $2-4 \mathrm{~nm}$ amorphous $\mathrm{SiO}_{x}$ interfacial layer. Secondary ion mass spectrometry (SIMS) of $\mathrm{SrTiO}_{3}$ on silicon is given in Supplementary Fig. 11, revealing carbon incorporation from the metalorganic precursor is limited primarily to the interface due to the decreased cracking efficiency of the metalorganic precursor at low temperatures. Carbon contamination is minimized for the bulk of the deposition at higher temperatures, in agreement with previous studies ${ }^{42}$.

\section{Discussion}

These growth experiments demonstrate that it is possible to integrate functional oxides in a scalable and economic way on $\mathrm{Si}$, addressing a long-standing challenge to synthesize high-quality perovskite materials on an industrial scale. The accessibility of the self-regulated growth window at high-growth rates afforded by hybrid MBE and the compatibility of this growth process with $\mathrm{Si}$ enables functional diversification of electronic devices in the current More than Moore era. The wide-ranging properties of perovskite oxides will open up routes to augment additional a

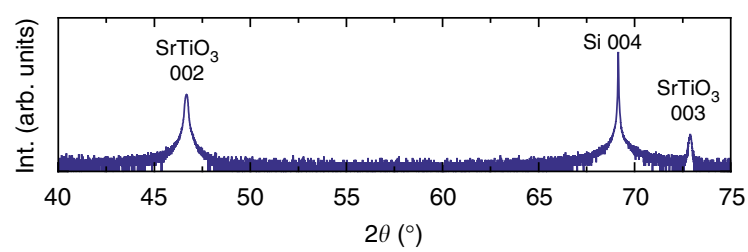

b

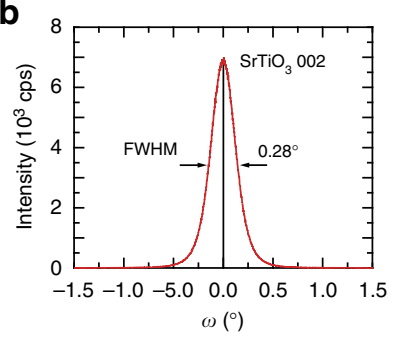

C
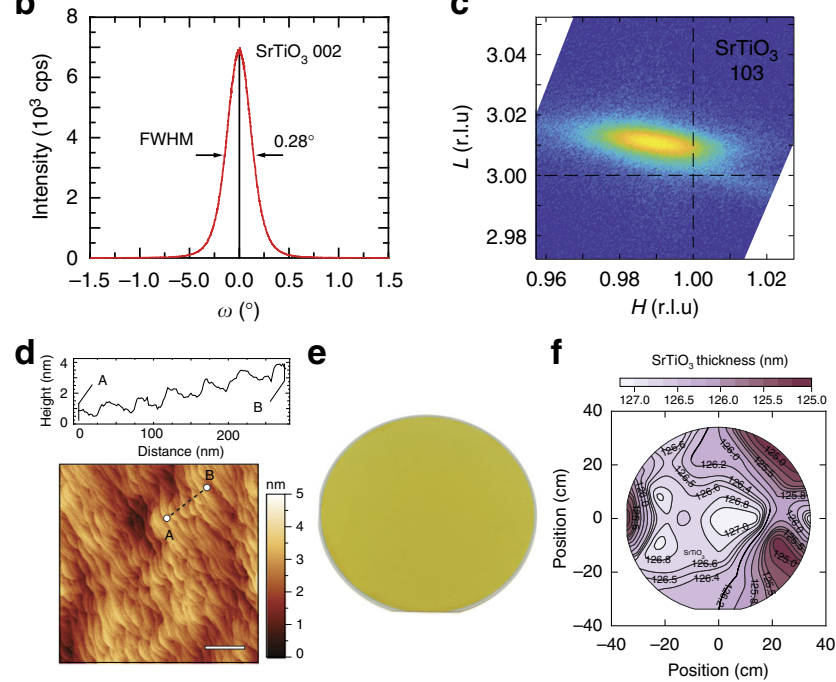

Fig. 5 Virtual perovskite oxide substrate on Si. a Wide range $2 \theta-\omega$ X-ray scan of a 126-nm-thick $\mathrm{SrTiO}_{3}$ film grown on 3 in $\mathrm{Si}$ wafer at a growth rate of $240 \mathrm{~nm} \mathrm{~h}^{-1}$. b Rocking curve ( $\omega$-scan) of the $002 \mathrm{SrTiO}_{3}$ peak with a full width at half maximum (FWHM) of $0.28^{\circ}$. c Reciprocal space map of the 103 $\mathrm{SrTiO}_{3}$ film peak in reciprocal lattice units of stoichiometric $\mathrm{SrTiO}_{3}$. d Atomic force microscopy image of the $\mathrm{SrTiO}_{3}$ on $\mathrm{Si}$ with "epi-ready" step terrace morphology and a surface roughness (RMS) of $\sim 1 \mathrm{~nm}$. A line profile of the film is shown above. Scale bar on AFM image is $200 \mathrm{~nm}$. e Photograph of the perovskite oxide virtual substrate, a $\mathrm{SrTiO}_{3}$ metamorphic buffer layer on a 3-in $\mathrm{Si}$ wafer. The $\mathrm{SrTiO}_{3}$ film is transparent, the yellow coloration is from interference due to its finite thickness $(\lambda / 4)$. $\mathbf{f}$ Wafer scale map of $\mathrm{SrTiO}_{3}$ film thickness on Si obtained from spectroscopic ellipsometry with a smaller than $1 \%$ film thickness variation across the wafer

functions to existing devices. The scalability of growth rates enables the development of a metamorphic buffer technology for virtual perovskite oxide substrates. Rather than being limited to single crystal substrates with sizes of up to only two inches using bulk crystal growth techniques such as Czochralski or Bridgman-Stockbarger methods, perovskite oxides on Si wafers with excellent crystalline quality and epi-ready surface morphologies provide more cost effective substrates and thus will enable more growth experiments. The availability of virtual substrates will drive innovation at the fundamental level and speed up the process development from basic scientific discoveries to system level maturity, thus boosting research productivity and technological significance of functional epitaxial oxide thin films. While accessing a self-regulated growth window at high growth rates was demonstrated for $\mathrm{SrTiO}_{3}$ as a prototypical perovskite oxide, many $\mathrm{ABO}_{3}$ perovskites $\left(\mathrm{ABO}_{3}, \mathrm{~A}=\mathrm{Ca}^{43}, \mathrm{Sr}^{40}, \mathrm{Ba}^{44}, \mathrm{Gd}^{45}, \mathrm{Nd}^{46}, \mathrm{Sm}^{47}\right.$ with $\mathrm{B}=\mathrm{Ti} ; \mathrm{A}=\mathrm{Sr}^{48}, \mathrm{Ca}^{49}, \mathrm{La}^{50}$ with $\mathrm{B}=\mathrm{V}$; as well as $\mathrm{LaAlO}_{3}{ }^{25}$, $\mathrm{BaSnO}_{3}{ }^{51}$, and $\mathrm{SrRuO}_{3}{ }^{52}$ ) have already been sucessfully grown by hybrid MBE. This suggests that scaling growth rates is a general feature of this growth approach rather than being limited to $\mathrm{SrTiO}_{3}$, indicating that the development of virtual substrates for perovskite oxides can be expanded to other perovskite oxides in an economic, scalable way as well. 


\section{Methods}

Thin film growth. $\mathrm{SrTiO}_{3}$ films were grown on $\mathrm{LSAT}, \mathrm{SrTiO}_{3}$, and $\mathrm{Si}$ substrates using a DCA M600 hMBE deposition system equipped with $2 \mathrm{Sr}$ dual filament solid source thermal effusion cells with $60 \mathrm{cc}$ crucibles. A gas delivery system was used to supply $\mathrm{Ti}$ in form of the volatile metalorganic molecule TTIP without a carrier gas. Sr fluxes were calibrated prior to growth using a quartz crystal monitor located at the sample growth position. The TTIP gas inlet pressure was monitored using a capacitance manometer in the injector system coupled to a linear leak valve to adjust and maintain the flux of TTIP to the substrate. Molecular oxygen was supplied and maintained at an oxygen background pressure of $\sim 3.0 \times 10^{-7}$ Torr. Growth windows were mapped out for fixed $\mathrm{Sr}$ fluxes between $1.25-25.0 \times 10^{13} \mathrm{~cm}^{-2} \mathrm{~s}^{-1}$, which were achieved by operating the $\mathrm{Sr}$ cells between 410 and $560^{\circ} \mathrm{C}$. A single $\mathrm{Sr}$ effusion cell was found to become unstable at fluxes exceeding $2.00 \times 10^{14} \mathrm{~cm}^{-2} \mathrm{~s}^{-1}$. Therefore, for high-growth rate experiments, a second $\mathrm{Sr}$ effusion cell was used in tandem to achieve a sufficiently large and stable flux. The reported growth rate was determined from film thickness measurements determined by fitting Laue oscillations of XRD data using GenX ${ }^{53}$. All $\mathrm{SrTiO}_{3}$ films on LSAT were grown at a temperature of $900{ }^{\circ} \mathrm{C}$ measured by the substrate heater thermocouple. $\mathrm{SrTiO}_{3}$ thin films were grown on Si wafers at $850^{\circ} \mathrm{C}$ using the process detailed in ref. ${ }^{35}$, followed by a post growth anneal, described in ref. ${ }^{37}$. Silicon wafers were etched in $10 \%$ buffered oxide etch, rinsed in deionised water, and placed under vacuum within $10 \mathrm{~min}$ of the initial etch. A one-half monolayer of $\mathrm{Sr}$ was deposited at $600^{\circ} \mathrm{C}$, followed by annealing at $720^{\circ} \mathrm{C}$ for $10 \mathrm{~min}$ to remove any additional oxygen before deposition. The sample was cooled to $400{ }^{\circ} \mathrm{C}$, and 4 monolayers of $\mathrm{SrTiO}_{3}$ were deposited by co-supplying $\mathrm{Sr}$ using the standard cell calibrated to flux of $2.5 \times$ $10^{13} \mathrm{~cm}^{-2} \mathrm{~s}^{-1}$ and TTIP at a baratron pressure of $52 \mathrm{mTorr}$ at a growth rate of $\sim 50 \mathrm{~nm} \mathrm{~h}^{-1}$. The temperature was then raised to $600^{\circ} \mathrm{C}$ and an additional 8 monolayers of $\mathrm{SrTiO}_{3}$ were deposited at the same fluxes to complete the buffer layer. The temperature was further increased to $850^{\circ} \mathrm{C}$ and a $10 \mathrm{~nm}$ layer of $\mathrm{SrTiO}_{3}$ was grown using the standard $\mathrm{Sr}$ flux and TTIP baratron pressure of $67 \mathrm{mT}$ orr to account for the increased desorption rate at elevated temperatures. Elemental oxygen was then introduced to the chamber during this stage to a background pressure of $3 \times 10^{-7}$ Torr. The second effusion cell calibrated to $1.0 \times 10^{14} \mathrm{~cm}^{-2} \mathrm{~s}^{-1}$ was used to deposit the remainder of the film a growth rate of $240 \mathrm{~nm} \mathrm{~h}^{-1}$, with a TTIP baratron pressure of 137 mTorr. After growth, samples were annealed in a furnace at $850^{\circ} \mathrm{C}$ in air to improve crystalline quality and relieve built-in strain in the films.

Atomic force microscopy. The surface morphology of $\mathrm{SrTiO}_{3}$ films grown at different growth rates on LSAT and Si were measured using a Bruker Dimension Icon atomic force microscope (AFM) operated in peak force tapping mode.

X-ray diffraction. High resolution of XRD of films grown within the growth window was carried out using a Phillips X'Pert Panalytical Pro operating with $\mathrm{Cu}$ $\mathrm{K}_{\alpha 1}$ radiation. $2 \theta-\omega$ scans were taken with an acceptance angle of $180^{\prime \prime}$, while rocking curve was recorded using a divergence angle of $26^{\prime \prime}$ an acceptance angle of $35^{\prime \prime}$. Reciprocal space map was taken on the same system with a divergence angle of $26^{\prime \prime}$, and recorded using a Panalytical Pixel3D detector.

Transmission electron microscopy. Cross-sectional high-resolution STEM was carried out using a FEI Titan operated at $200 \mathrm{kV}$ accelerating voltage. TEM sample preparation of films on LSAT substrates consisted of mechanical polishing at wedge angle of $2^{\circ}$ to a thickness of $<5 \mu \mathrm{m}$, followed by thinning to electron transparency using a Gatan PIPSII Ar-ion milling along [100]. Samples on silicon were prepared using the Scios DualBeam focused ion beam using $\mathrm{Ga}^{+}$ions. Accelerating voltage was scaled from $30 \mathrm{keV}$ for the initial lamella preparation, and scaled down to $2 \mathrm{keV}$ for the final sample thinning.

Spectroscopic ellipsometry. Ellipsometric data, i.e., phase difference $\Delta$ and amplitude ratio $\Psi$ in the spectral range from $0.75-3.0 \mathrm{eV}$ were collected from 25 different locations across the wafer (see details in Supplementary Fig. 8) using a J. A. Woollam Co., Inc. M-2000XI single rotating compensator spectroscopic ellipsometer in reflection mode at 3 different angles of incidence, $50^{\circ}, 60^{\circ}$, and $70^{\circ}$. The thickness values and refractive index for the $\mathrm{SrTiO}_{3}$ film were extracted by means of a least squares regression analysis employing the Levenberg-Marquardt algorithm. An optical model consisting of a Si substrate (semi-infinite)/native silicon oxide interface layer $/ \mathrm{SrTiO}_{3}$ film $/ \mathrm{SrTiO}_{3}$ film surface roughness was used to extract the physical parameters. The surface roughness was represented by a Bruggeman effective medium approximation of 0.5 void +0.5 film material fractions. For the substrate and silicon native oxide layer, the silicon ${ }^{54}$ and silicon dioxide $^{54}$ material parameters were used. The parameterization of the refractive index of the $\mathrm{SrTiO}_{3}$ film included a combination of the high-frequency dielectric constant and two Sellmeier oscillators. Oscillator parameters as well as thicknesses of the $\mathrm{SrTiO}_{3}$ film, the native oxide film, and the surface roughness layer were used as fitting parameters.
Secondary ion mass spectrometry. SIMS of $\mathrm{SrTiO}_{3}$ on silicon was performed using a PHI nano TOF. A primary $30 \mathrm{keV} \mathrm{Bi}_{3}{ }^{+}$ion beam was rastered over an area of $100 \times 100 \mu \mathrm{m}$. The etch rate was approximately $3 \mathrm{~nm}$ cycle $^{-1}$.

\section{Data availability}

The data that support the findings of this study are available from the corresponding author upon reasonable request.

Received: 24 October 2018 Accepted: 12 April 2019

Published online: 05 June 2019

\section{References}

1. Kang, K. et al. High-mobility three-atom-thick semiconducting films with wafer-scale homogeneity. Nature 520, 656-660 (2015).

2. France, R. M. et al. Design flexibility of ultrahigh efficiency four-junction inverted metamorphic solar cells. IEEE J. Photovolt. 6, 578-583 (2016).

3. Dimroth, F. et al. Four-junction wafer-bonded concentrator solar cells. IEEE J. Photovolt. 6, 343-349 (2016).

4. Green, M. A. et al. Solar cell efficiency tables (version 49). Prog. Photovolt. Res. Appl. 25, 3-13 (2017).

5. Tournié, E. et al. Metamorphic III-V semiconductor lasers grown on silicon MRS Bull. 41, 218-223 (2016).

6. Cerba, T. et al. Anti phase boundary free GaSb layer grown on $300 \mathrm{~mm}(001)$ Si substrate by metal organic chemical vapor deposition. Thin Solid Films $\mathbf{6 4 5}$, 5-9 (2018).

7. Bennett, B. R., Magno, R., Boos, J. B., Kruppa, W. \& Ancona, M. G. Antimonide-based compound semiconductors for electronic devices: a review. Solid. State Electron. 49, 1875-1895 (2005).

8. Lee, K. E. \& Fitzgerald, E. A. Metamorphic transistors: building blocks for hetero-integrated circuits. MRS Bull. 41, 210-217 (2016).

9. Ng, G. I., Radhakrishnan, K. \& Wang, H. Are we there yet?-a metamorphic HEMT and HBT perspective. Eur. Gall. Arsenide Other Semicond. Appl. Symp. GAAS 2005, 13-19 (2005).

10. Fathpour, S. Emerging heterogeneous integrated photonic platforms on silicon. Nanophotonics 4, 143-164 (2015).

11. Tian, P. et al. Characteristics and applications of micro-pixelated GaN-based light emitting diodes on Si substrates. J. Appl. Phys. 115, 033112 (2014).

12. Weiss, B. et al. Monolithically-integrated mulitlevel inverter on lateral GaNon-Si technology for high-voltage applications. In 2015 IEEE Compound Semiconductor Integrated Circuit Symposium (CSICS) 1-4 (IEEE, New Orleans, LA, 2015).

13. Eng, K. et al. Isotopically enhanced triple-quantum-dot qubit. Sci. Adv. 1, e1500214-e1500214 (2015).

14. Schlom, D. G. et al. Strain tuning of ferroelectric thin films. Annu. Rev. Mater Res. 37, 589-626 (2007).

15. McKee, R. A., Walker, F. J. \& Chisholm, M. F. Crystalline oxides on silicon: the first five monolayers. Phys. Rev. Lett. 81, 3014-3017 (1998).

16. Gu, X. et al. Growth, characterization, and uniformity analysis of $200 \mathrm{~mm}$ wafer-scale $\mathrm{SrTiO}_{3} /$ Si. J. Vac. Sci. Technol. B 28, C3A12-C3A16 (2010).

17. Brooks, C. M. et al. Growth of homoepitaxial $\mathrm{SrTiO}_{3}$ thin films by molecularbeam epitaxy. Appl. Phys. Lett. 94, 162905 (2009).

18. Yamaguchi, H., Matsubara, S. \& Miyasaka, Y. Reactive coevaporation synthesis and characterization of $\mathrm{SrTiO}_{3}$ thin films. Jpn. J. Appl. Phys. 30, 2197-2199 (1991).

19. Fisher, P. et al. Stoichiometric, nonstoichiometric, and locally nonstoichiometric $\mathrm{SrTiO}_{3}$ films grown by molecular beam epitaxy. J. Appl. Phys. 103, 013519 (2008).

20. Cho, A. Y. How molecular beam epitaxy (MBE) began and its projection into the future. J. Cryst. Growth 201, 1-7 (1999).

21. Sonoda, T. et al. Ultra-high throughput of GaAs and (AlGa)As layers grown by MBE with a specially designed MBE system. J. Cryst. Growth 95, 317-321 (1989).

22. Heyn, C., Franke, T., Anton, R. \& Harsdorff, M. Correlation between islandformation kinetics, surface roughening, and RHEED oscillation damping during GaAs homoepitaxy. Phys. Rev. B 56, 13483-13489 (1997).

23. Henini, M. Molecular Beam Epitaxy from Research to Mass Production. (Elsevier, Waltham, MA, USA, 2012).

24. Richardson, C. J. K. \& Lee, M. L. Metamorphic epitaxial materials. MRS Bull. 41, 193-198 (2016).

25. Brahlek, M. et al. Frontiers in the growth of complex oxide thin films: past, present, and future of hybrid MBE. Adv. Funct. Mater. 28, 1702772 (2018).

26. Jalan, B., Moetakef, P. \& Stemmer, S. Molecular beam epitaxy of $\mathrm{SrTiO}_{3}$ with a growth window. Appl. Phys. Lett. 95, 032906 (2009).

27. Ambwani, P. et al. Defects, stoichiometry, and electronic transport in $\mathrm{SrTiO}_{3-\delta}$ epilayers: a high pressure oxygen sputter deposition study. J. Appl. Phys. 120 055704 (2016). 
28. Ohnishi, T., Lippmaa, M., Yamamoto, T., Meguro, S. \& Koinuma, H. Improved stoichiometry and misfit control in perovskite thin film formation at a critical fluence by pulsed laser deposition. Appl. Phys. Lett. 87, 241919 (2005).

29. Greer, J. A. History and current status of commercial pulsed laser deposition equipment. J. Phys. D. Appl. Phys. 47, 034005 (2014).

30. Arai, T. T., Inaishi, Y., Sawado, Y., Kobayashi, I. \& Hidaka, J. Preparation of $\mathrm{SrTiO}_{3}$ films on 8-inch wafers by chemical vapor deposition. Jpn. J. Appl. Phys. 35, 4875-4879 (1996).

31. Kim, Y.-S., Bansal, N. \& Oh, S. Simple self-gettering differential-pump for minimizing source oxidation in oxide-MBE environment. J. Vac. Sci. Technol. A Vacuum. Surf., Film. 29, 041505 (2011).

32. Haeni, J. H. H., Theis, C. D. D. \& Schlom, D. G. G. RHEED Intensity Oscillations for the Stoichiometric Growth of $\mathrm{SrTiO}_{3}$ Thin Films by Reactive Molecular Beam Epitaxy. J. Electroceram. 4, 385-391 (2000).

33. Li, H. et al. Two-dimensional growth of high-quality strontium titanate thin films on Si. J. Appl. Phys. 93, 4521-4525 (2003).

34. Brahlek, M., Zhang, L., Eaton, C., Zhang, H.-T. \& Engel-Herbert, R. Accessing a growth window for $\mathrm{SrVO}_{3}$ thin films. Appl. Phys. Lett. 107, 143108 (2015).

35. Zhang, L. et al. Continuously tuning epitaxial strains by thermal mismatch. ACS Nano 12, 1306-1312 (2018).

36. Kajdos, A. P. \& Stemmer, S. Surface reconstructions in molecular beam epitaxy of SrTiO3. Appl. Phys. Lett. 105, 191901 (2014).

37. Zhang, L., Wang, Y. \& Engel-Herbert, R. Improving the structural quality and electrical resistance of $\mathrm{SrTiO} 3$ thin films on $\mathrm{Si}(001)$ via a two-step anneal. J. Appl. Phys. 119, 045301 (2016).

38. Zhang, L. \& Engel-Herbert, R. Growth of $\mathrm{SrTiO}_{3}$ on $\mathrm{Si}(001)$ by hybrid molecular beam epitaxy. Phys. Status Solidi 8, 917-923 (2014).

39. Wang, T., Ganguly, K., Marshall, P., Xu, P. \& Jalan, B. Critical thickness and strain relaxation in molecular beam epitaxy-grown $\mathrm{SrTiO}_{3}$ films. Appl. Phys. Lett. 103, 212904 (2013).

40. Jalan, B., Engel-Herbert, R., Wright, N. J. \& Stemmer, S. Growth of highquality $\mathrm{SrTiO}_{3}$ films using a hybrid molecular beam epitaxy approach. J. Vac. Sci. Technol. A 27, 461 (2009).

41. LeBeau, J. M. et al. Stoichiometry optimization of homoepitaxial oxide thin films using x-ray diffraction. Appl. Phys. Lett. 95, 142905 (2009).

42. Jalan, B., Cagnon, J., Mates, T. E. \& Stemmer, S. Analysis of carbon in SrTiO3 grown by hybrid molecular beam epitaxy. J. Vac. Sci. Technol. A 27, 1365-1368 (2009).

43. Haislmaier, R. C. et al. Unleashing strain induced ferroelectricity in complex oxide thin films via precise stoichiometry control. Adv. Funct. Mater. 26, 7271-7279 (2016).

44. Matsubara, Y., Takahashi, K. S., Tokura, Y. \& Kawasaki, M. Single-crystalline $\mathrm{BaTiO}_{3}$ films grown by gas-source molecular beam epitaxy. Appl. Phys. Express 7, 125502 (2014).

45. Zhang, J. Y., Jackson, C. A., Raghavan, S., Hwang, J. \& Stemmer, S. Magnetism and local structure in low-dimensional Mott insulating GdTiO3. Phys. Rev. B 88, 121104 (2013).

46. Xu, P., Phelan, D., Jeong, J. S., Mkhoyan, K. A. \& Jalan, B. Stoichiometrydriven metal-to-insulator transition in $\mathrm{NdTiO}_{3} / \mathrm{SrTiO}_{3}$ heterostructures. Appl. Phys. Lett. 104, 082109 (2014).

47. Ahadi, K. \& Stemmer, S. Novel metal-insulator transition at the $\mathrm{SmTiO}_{3}$ $\mathrm{SrTiO}_{3}$ interface. Phys. Rev. Lett. 118, 236803 (2017)

48. Eaton, C. et al. Growth of $\mathrm{SrVO}_{3}$ thin films by hybrid molecular beam epitaxy. J. Vac. Sci. Technol. A 33, 061504 (2015).

49. Eaton, C., Lapano, J., Zhang, L., Brahlek, M. \& Engel-Herbert, R. Selfregulated growth of $\mathrm{CaVO}_{3}$ by hybrid molecular beam epitaxy. J. Vac. Sci. Technol. A 35, 061510 (2017).

50. Zhang, H. -T., Dedon, L. R., Martin, L. W. \& Engel-Herbert, R. Self-regulated growth of $\mathrm{LaVO}_{3}$ thin films by hybrid molecular beam epitaxy. Appl. Phys. Lett. 106, 233102 (2015).
51. Prakash, A. et al. Hybrid molecular beam epitaxy for the growth of stoichiometric $\mathrm{BaSnO}_{3}$. J. Vac. Sci. Technol. A 33, 060608 (2015).

52. Marshall, P. B., Kim, H., Ahadi, K. \& Stemmer, S. Growth of strontium ruthenate films by hybrid molecular beam epitaxy. APL Mater. 5, 096101 (2017).

53. Björck, M. \& Andersson, G. \& IUCr. GenX: an extensible X-ray reflectivity refinement program utilizing differential evolution. J. Appl. Crystallogr. 40, 1174-1178 (2007)

54. Palik, E. D., Philipp, H. R. \& Aspnes, D. E. Handbook of Optical Constants of Solids. (Academic Press, San Diego, California, 1985).

\section{Acknowledgements}

J.M.L. and R.E.H. acknowledge National Science Foundation through the Penn State MRSEC program DMR-1420620, J.R. acknowledges DMR-1629477 and support through the NSF graduate student fellowship, M.B. and R.E.H. acknowledge the Department of Energy (Grant DE-SC0012375), L.Z. acknowledges the National Science Foundation through DMR-1352502. We thank Dr. Arnab Sen Gupta for assisting in growth of samples, Profs. Jon-Paul Maria and Venkat Gopalan, as well as Drs. Craig Eaton and Julian Walker for helpful discussions.

\section{Author contributions}

J.L., M.B., L.Z. and R.E.H. conceived the project. J.L., J.R. and M.B. performed the growth and characterization of $\mathrm{SrTiO}_{3}$ films on LSAT, and $\mathrm{SrTiO}_{3}$ at high-growth rates, as well as XRD and AFM characterization of all samples. J.L. and L.Z. grew $\mathrm{SrTiO}_{3}$ films on silicon. A.P. measured the film thickness using spectroscopic ellipsometry, J.L. prepared and imaged the HR-STEM samples. J.L. and R.E.H. co-wrote the paper with input and suggestions from all authors.

\section{Additional information}

Supplementary Information accompanies this paper at https://doi.org/10.1038/s41467019-10273-2.

Competing interests: The authors declare no competing interests.

Reprints and permission information is available online at http://npg.nature.com/ reprintsandpermissions/

Journal peer review information: Nature Communications thanks the anonymous reviewer(s) for their contribution to the peer review of this work. Peer reviewer reports are available.

Publisher's note: Springer Nature remains neutral with regard to jurisdictional claims in published maps and institutional affiliations.

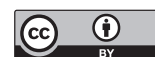

Open Access This article is licensed under a Creative Commons Attribution 4.0 International License, which permits use, sharing, adaptation, distribution and reproduction in any medium or format, as long as you give appropriate credit to the original author(s) and the source, provide a link to the Creative Commons license, and indicate if changes were made. The images or other third party material in this article are included in the article's Creative Commons license, unless indicated otherwise in a credit line to the material. If material is not included in the article's Creative Commons license and your intended use is not permitted by statutory regulation or exceeds the permitted use, you will need to obtain permission directly from the copyright holder. To view a copy of this license, visit http://creativecommons.org/ licenses/by/4.0/.

(c) The Author(s) 2019 